\title{
Aspectos relacionados à administração e conservação de vacinas em centros de saúde no Nordeste do Brasil
}

\author{
Aspects related to vaccine management and preservation \\ in healthcare centers in the Northeastern Brazil
}

Geisy Lanne Muniz Luna ${ }^{1}$

Luiza Jane Eyre de Souza Vieira ${ }^{1}$

Priscilla Freire de Souza ${ }^{1}$

Samira Valentim Gama Lira ${ }^{1}$

Deborah Pedrosa Moreira ${ }^{1}$

Aline de Souza Pereira ${ }^{1}$

${ }^{1}$ Programa de Mestrado em Saúde Coletiva, Centro de Ciências da Saúde,

Universidade de Fortaleza. Av. Washington Soares 1.321, Bairro Edson Queiroz. 60811-905 Fortaleza CE. geisylanne@hotmail.com ${ }^{2}$ Prefeitura de Fortaleza (CE).

${ }^{3}$ Escola de Saúde Pública do Ceará.

\begin{abstract}
This study characterizes the expertise and updated knowledge on immunization of nursing team responsible for vaccine rooms; it describes the work conditions in that sector from the team's view, and identifies the procedures and daily activities performed by such professionals. This cross-sectional study conducted in 2007 in vaccine rooms of 11 Family Healthcare Centers (CSF) in Fortaleza (CE, Brazil), involved 22 nursing team professionals and used a checklist and selfapplicable questionnaire as the survey instrument. Among the participants, $44.4 \%$ were nurses and $30.8 \%$ technicians/assistants have an experience of more than five years; $55.6 \%$ of the nurses had attended no training on vaccines; and $76.9 \%$ of all other professionals keep themselves updated by referring to books, manuals and the Internet. Gaps in physical structure, examination and record of temperature maps, and guidance on post-vaccine effects were detected in most rooms under study. The study indicates that managing any vaccination work requires knowledge of the area and specific training to allow the professionals to provide a quality service to the population in a way to ensure the eradication and control of immunopreventable diseases.
\end{abstract}

Key words Immunization, Vaccines, Nursing team, Cold chain
Resumo O estudo caracteriza a experiência e atualização do conhecimento sobre imunização da equipe de enfermagem responsável pelas salas de vacinas, descreve as condições de trabalho nesse setor a partir da visão dessa equipe e identifica os procedimentos e atividades diárias realizados por esses profissionais. Trata-se de estudo transversal, realizado em 2007, nas salas de vacinas de 11 Centros de Saúde da Família (CSF) em Fortaleza (CE), com a participação de 22 profissionais da equipe de enfermagem. Utilizou check-list e questionário autoaplicável como instrumentos de coleta. Entre as participantes, 44,4\% das enfermeiras e 30,8\% dos técnicos/auxiliares têm mais de cinco anos de experiência; 55,6\% das enfermeiras não possuíam treinamento em vacinas; $88,9 \%$ das enfermeiras e $76,9 \%$ dos outros profissionais se mantêm atualizados consultando livros, manuais e Internet. Detectaram-se lacunas quanto a estrutura física, verificação e registro do mapa de temperatura e orientações sobre os efeitos pósvacinais na maioria das salas estudadas. O estudo sinaliza que gerenciar o trabalho com imunobiológicos requer conhecimento e treinamento específico para que se possa ofertar serviço de qualidade e que não comprometa a erradicação e o controle de doenças imunopreveníveis.

Palavras-chave Imunização, Vacinas, Equipe de enfermagem, Cadeia de frio 


\section{Introdução}

A literatura reconhece a importância dos programas de imunização como uma das medidas mais eficazes para a promoção da saúde dos povos ${ }^{1,2}$, independentemente das diferenças socioeconômicas e culturais. Alerta que o desenvolvimento de vacinas seguras e efetivas para a prevenção de doenças infecciosas, geralmente associadas com alta mortalidade e morbidade, é uma das mais relevantes ações da tecnologia em saúde $\mathrm{e}^{3-5}$.

O Programa Nacional de Imunizações (PNI) ${ }^{6}$ é referência mundial para a organização de campanhas de vacinas em países como Timor Leste e auxilia nos programas de imunizações na Palestina, na Cisjordânia e na Faixa de Gaza, estabelecendo cooperação técnica em outros países. Ações planejadas e sistematizadas desenvolvidas em nosso país erradicaram a varíola em 1973 e a poliomielite em 1989, e mantém sob monitoração as doenças imunopreveníveis da infância ${ }^{7}$. O PNI está vigilante ao perfil epidemiológico das doenças infectocontagiosas e tem ampliado a oferta de imunobiológicos, tanto em vacinações de bloqueio como em seu próprio calendário básico ${ }^{8,9}$, o que repercute para a melhoria da saúde brasileira.

Importante mencionar a resolubilidade do Ministério da Saúde ${ }^{9}$ diante da epidemia mundial da Gripe $\mathrm{H}_{1} \mathrm{~N}_{1}$, distribuindo a vacina viral pandêmica para os grupos suscetíveis aos casos graves e à letalidade ${ }^{9,10}$, em nível nacional, iniciada em março de $2010^{9}$. Seguindo outros países ${ }^{1,2}$, o Brasil também amplia a cobertura vacinal da hepatite B para gestantes após o primeiro trimestre, manicures, podólogos e tatuadores ${ }^{11,12}$.

Firmando-se como um dos pilares dos cuidados primários em saúde nos países desenvolvidos e em desenvolvimento ${ }^{13}$, no Brasil a Estratégia Saúde da Família é o campo profícuo para operacionalizar ações de educação e promoção da saúde, demonstrando à sociedade as evidências dos benefícios da imunização ${ }^{14}$ para o alcance de metas preconizadas pelos órgãos internacionais ${ }^{15}$. No intuito de sistematizar a atenção básica a Norma Operacional de Assistência à Saúde (Noas $)^{16}$, definiu responsabilidades e estratégicas mínimas que os municípios deviam ofertar à população, no âmbito da saúde da criança, da mulher e saúde bucal; controle da tuberculose, hipertensão arterial e diabetes mellitus; eliminação da hanseníase ${ }^{17}$. Na saúde da criança são áreas prioritárias a vigilância nutricional, imunização, assistência às doenças prevalentes na infância e assistência e prevenção às patologias bucais ${ }^{18}$.

Reforçando as diretrizes da Noas ${ }^{16}$, o pacto pela saúde ${ }^{18}$ enfatiza a necessidade de se reduzir a mortalidade infantil, e esta redução associa-se com menor incidência das doenças imunopreveníveis. Nessa perspectiva, para o êxito dos programas de vacinação, não são suficientes a segurança e a eficácia dos imunobiológicos se os profissionais de saúde não os operacionalizarem de acordo com as recomendações específicas (conservação, manipulação, administração, acompanhamento pós-vacinal, dentre outras) ou se a população não aderir à vacinação $0^{3,6}$.

A literatura aponta ${ }^{1,19,20}$ que entre os profissionais da Estratégia Saúde da Família a equipe de enfermagem é a responsável pelo gerenciamento e oferta de imunobiológicos à população adscrita, o que nos remete à necessidade de acompanhar o processo de trabalho nas salas de vacinas e o posicionamento desses profissionais sobre o trabalho desenvolvido.

Assim, este estudo: (1) caracteriza a experiência e atualização do conhecimento sobre imunização da equipe de enfermagem responsável pelas atividades nas salas de vacinas; (2) descreve as condições de trabalho nesse setor a partir da visão da equipe de enfermagem; e (3) identifica a prática de higienização manual e orientações fornecidas ao usuário.

\section{Material e métodos}

Trata-se de um estudo transversal, realizado em 11 (92\%) dos 12 Centros de Saúde da Família (CSF) da Secretaria Executiva Regional IV, em Fortaleza, Ceará, em 2007. O município de Fortaleza possui uma área de 313,8 quilômetros quadrados, uma população de 2.431.415 habitantes $^{21}$, com 234.674 crianças entre zero e quatro anos; apresenta uma mortalidade infantil de 15,5 por mil nascidos vivos, considerando apenas os óbitos e nascimentos coletados pelo Sistema de Informação sobre Mortalidade e pelo Sistema de Informação de Nascidos Vivos (SIM/Sinasc) ${ }^{22}$.

O relatório "O estado das cidades do mundo 2010/2011: unindo o urbano dividido”, da Organização das Nações Unidas ${ }^{23}$, destacou Fortaleza entre as quatro cidades mais desiguais do Brasil, seguida de Goiânia, Belo Horizonte e Brasília. Estas cidades apresentam o Índice de Gini (índice de desigualdade medido em uma intervalo de 0 a 1 , tendo como base o consumo) superior a 0,60 ; quanto mais próximo de 1 , maior a desigualdade entre o que as pessoas ganham.

Atualmente com 114 bairros, estes se agrupam em seis “Secretarias Executivas Regionais” (SERs), unidades administrativas diretas da prefeitura, responsáveis pela execução dos serviços públicos nas 
áreas delimitadas. A SER não tem área sobreposta à dos distritos históricos, que não têm função administrativa, mas as sedes são próximas aos núcleos das seis secretarias ${ }^{24}$. A área administrativa sede do presente trabalho, inclui 19 bairros, conta com 12 Centros de Saúde que atendem na lógica da Estratégia Saúde da Família.

Sabe-se que a equipe de enfermagem é responsável pelo gerenciamento e administração de vacinas $^{1,19,20}$, em países desenvolvidos ${ }^{1}$ e em desenvolvimento ${ }^{19}$. O estudo elegeu esses profissionais como população/amostra, estabelecendo como critérios de inclusão: (1) pertencer à equipe de enfermagem da unidade de saúde; (2) trabalhar em salas de vacinas; e (3) dispor-se a participar do estudo, sem qualquer objeção. Atendendo aos critérios, participaram do estudo 22 profissionais, enfermeiras (nove), técnicos e auxiliares (treze), o que correspondeu a $19,3 \%$ da equipe de enfermagem da Regional IV.

Questionário autoaplicável $(n=22)$ e preenchimento de um check-list $(\mathrm{n}=11)$ pela observação direta foram os instrumentos de coleta dos dados. O questionário abordou as características da equipe de enfermagem quanto a: idade, tempo de trabalho na atenção básica, experiência de trabalho com vacinas, justificativa para o profissional ter sido designado para este setor, possuir treinamento em imunobiológicos, de que maneira se mantém atualizado sobre o tema, conservação dos imunobiológicos de acordo com as recomendações técnicas do PNI e a prática de busca ativa na atualização do calendário vacinal.

O check-list permitiu identificar aspectos da estrutura física da sala (decoração, localização, iluminação, climatização), anotações do mapa diário de temperatura, higienização das mãos pelas profissionais, explicações sobre o procedimento e orientações sobre os efeitos pós-vacinais aos usuários.

A coleta de dados transcorreu entre agosto e setembro de 2007, em dias previamente agendados, alternando-se entre os turnos matutinos e vespertinos, mediante ciência e deliberação dos gestores dos CSF e das participantes da pesquisa, e realizada por uma das autoras. $\mathrm{Na}$ organização, codificação e análise estatística descritiva com o emprego de testes paramétricos, cálculo das frequências absolutas e percentuais e medidas de tendência central, utilizou-se o Programa Statistical Package Social Science (SPSS), versão 13.0 for Windows (SPSS Inc. Chicago, USA). Estes foram discutidos conforme a literatura pertinente ao tema, munindo-se também de documentos oficiais.
O estudo foi aprovado pelo Comitê de Ética e Pesquisa da Universidade de Fortaleza (Unifor) e autorizado pela Secretaria de Saúde do Município de Fortaleza (SMS).

\section{Resultados}

Sobre a equipe de enfermagem responsável pelas salas de imunização dos CSF (Regional IV), a Tabela 1 mostra os resultados da variável faixa etária, da experiência de trabalho na atenção básica e no trabalho com vacinas, o modo como a profissional foi designada para trabalhar no setor de imunização, se possui treinamento sobre o tema e quais as estratégias para se manter atualizada sobre o assunto (Tabela 1).

A equipe de enfermagem deste estudo, 100\% de mulheres, confirma a predominância feminina da profissão $0^{1,19}$. A idade média entre as enfermeiras foi de 36,4 anos, variando de 26 a 46 anos; entre as técnicas e as auxiliares, a idade média foi de 37,6 anos, no intervalo de 19 a 48 anos.

Entre as respondentes $(n=22), 83,3 \%$ relataram que os profissionais do CSF encaminham o usuário para a sala de vacina; em contrapartida, a busca ativa de faltosos, identificada em apenas $16,7 \%$, ainda não foi incorporada por alguns profissionais $(83,3 \%)$.

A visão da equipe de enfermagem sobre as condições e dinâmica de trabalho nas salas de vacinas quanto ao suprimento de material (os próprios imunobiológicos, seringas, agulhas, algodão, material de escritório etc.) para atender a demanda, disponibilidade de literatura nas salas para atualização em imunobiológicos, se a sala é exclusiva para as atividades de imunização, se o usuário enfrenta fila para se vacinar e se este realiza cadastro neste setor estão demonstrados na Tabela 2 .

Quando se analisaram comparativamente as respostas das enfermeiras com as emitidas pelas técnicas/auxiliares, quanto à existência do material de pesquisa/atualização disponível nas salas de imunização, esses dados divergiram, pois $100 \%$ das enfermeiras confirmaram a disponibilidade desse material, enquanto 15,4\% de técnicas/auxiliares negaram a existência desse recurso. Merecem atenção as respostas da equipe de enfermagem, constando que três $(13,6 \%)$ negaram que a sala de vacina seja exclusiva para realizar vacinação (Tabela 2).

A Tabela 3 mostra alguns aspectos inerentes à rotina das salas de imunização: a higiene das mãos por parte do profissional que está admi- 
Tabela 1. Caracterização da equipe de enfermagem que trabalha nas salas de vacinas. Fortaleza, Ceará, 2007 $(\mathrm{n}=22)$.

\begin{tabular}{|c|c|c|c|c|}
\hline \multirow[t]{2}{*}{ Características } & \multicolumn{2}{|c|}{ Enfermeiro $(n=09)$} & \multicolumn{2}{|c|}{ Técnicos/auxiliares $(n=13)$} \\
\hline & $\mathrm{n}$ & $\%$ & $\mathrm{n}$ & $\%$ \\
\hline \multicolumn{5}{|l|}{ Idade (anos) } \\
\hline $18 \mid-25$ & - & - & 02 & 15,4 \\
\hline $25 \mid-30$ & 01 & 11,1 & 04 & 30,8 \\
\hline $30 \mid-40$ & 05 & 55,6 & 04 & 30,8 \\
\hline$>40$ & 03 & 33,3 & 03 & 23,0 \\
\hline \multicolumn{5}{|l|}{ Experiência de trabalho na $\mathrm{AB}^{*}$ (anos) } \\
\hline Menos de 01 & - & - & 03 & 23,0 \\
\hline 01 a 03 & 01 & 11,1 & 05 & 38,5 \\
\hline 04 a 05 & 01 & 11,1 & - & - \\
\hline Mais de 05 & 07 & 77,8 & 05 & 38,5 \\
\hline \multicolumn{5}{|l|}{ Experiência no trabalho com vacinas (anos) } \\
\hline Menos de 01 & 01 & 11,1 & 04 & 30,8 \\
\hline 01 a 03 & 02 & 22,2 & 05 & 38,4 \\
\hline 03 a 05 & 02 & 22,2 & - & - \\
\hline Mais de 05 & 04 & 44,4 & 04 & 30,8 \\
\hline \multicolumn{5}{|l|}{ Designação para o setor } \\
\hline Afinidade & 02 & 22,2 & 02 & 15,3 \\
\hline Capacitação na área & 01 & 11,1 & 05 & 38,5 \\
\hline Experiência & 02 & 22,2 & 01 & 7,7 \\
\hline Indicação da chefia & 03 & 33,3 & 05 & 38,5 \\
\hline Outros & 01 & 11,1 & 00 & - \\
\hline \multicolumn{5}{|l|}{ Treinamento sobre imunização } \\
\hline Sim & 04 & 44,4 & 10 & 77,0 \\
\hline Não & 05 & 55,6 & 03 & 23,0 \\
\hline \multicolumn{5}{|l|}{ Como se mantém atualizado sobre o tema } \\
\hline Materiais disponíveis na sala & 01 & 11,1 & 02 & 15,4 \\
\hline Livros, manuais e consulta à Internet & 08 & 88,9 & 10 & 76,9 \\
\hline Não se atualiza & - & - & 01 & 7,7 \\
\hline \multicolumn{5}{|l|}{ Tipo de material para atualização } \\
\hline Ministério da Saúde (MS) & 08 & 88,9 & 10 & 83,3 \\
\hline Secretaria Municipal Saúde (SMS) & 01 & 11,1 & 01 & 16,7 \\
\hline
\end{tabular}

Fonte: Pesquisa direta, 2007.

*Atenção Básica.

nistrando a vacina, explicação do procedimento (administração de imunobiológicos e sua respectiva indicação) ao usuário e/ou aos seus familiares (mães/pais/avós) e explicação dos efeitos pósvacinais, como preconiza o Manual de efeitos adversos pós-vacinação $o^{25}$.

No período de visitas/observação das salas de vacinas dos CSF $(n=11)$ durante a aplicação de imunobiológicos, constatou-se que, apesar de todas as salas disporem da estrutura para o profissional realizar a higiene das mãos (pia, sabão e toalha descartável ou de tecido), 8,3\% das responsáveis pela administração das vacinas não higienizaram as mãos. Contrapondo-se a essa observação, em $72,7 \%$ das salas as profissionais explicaram o procedimento a ser realizado; contudo, as orientações sobre as possíveis reações pós-vacinais não foram detectadas, registrando o mesmo percentual anterior em $72,7 \%$ das salas de vacinas observadas.

Durante as visitas (observação e check-list), verificou-se que as geladeiras em uso eram especificadas como domésticas, com capacidade média entre 280 a 300 litros, atendendo às recomendações do Ministério da Saúde ${ }^{26}$, com tempo médio de uso entre um e dois anos, e todos os CSF tinham equipamentos para conservar as vacinas no caso dos desligamentos programados (degelo da geladeira) e/ou panes na rede elé- 
Tabela 2. Visão da equipe de enfermagem sobre as condições e a dinâmica de trabalho nas salas de vacinas. Fortaleza, Ceará, $2007(\mathrm{n}=22)$.

\begin{tabular}{|c|c|c|c|c|}
\hline & \multicolumn{2}{|c|}{ Enfermeiro $(n=09)$} & \multicolumn{2}{|c|}{ Técnicos/auxiliares $(n=13)$} \\
\hline & $\mathrm{n}$ & $\%$ & $\mathrm{n}$ & $\%$ \\
\hline \multicolumn{5}{|c|}{ Material suficiente } \\
\hline Sim & 08 & 88,9 & 11 & 84,6 \\
\hline Não & 01 & 11,1 & 02 & 15,4 \\
\hline \multicolumn{5}{|c|}{ Literatura* disponível na sala } \\
\hline Sim & 09 & 100 & 11 & 84,6 \\
\hline Não & - & - & 02 & 15,4 \\
\hline \multicolumn{5}{|c|}{ Sala exclusiva para vacinação } \\
\hline Sim & 08 & 88,9 & 11 & 84,6 \\
\hline Não & 01 & 11,1 & 02 & 15,4 \\
\hline \multicolumn{5}{|c|}{ Usuário enfrenta fila para se vacinar } \\
\hline Sim & 05 & 55,6 & 09 & 69,2 \\
\hline Não & 04 & 44,4 & 04 & 30,8 \\
\hline \multicolumn{5}{|c|}{ Usuário é cadastrado na sala de vacina } \\
\hline Sim & 03 & 33,3 & 07 & 53,8 \\
\hline Não & 06 & 66,6 & 06 & 46,2 \\
\hline
\end{tabular}

Fonte: Pesquisa direta, 2007.

*Livros, manuais, informes técnicos, publicações on-line etc.

Tabela 3. Procedimentos e atividades diárias realizados pelos profissionais nas salas de vacinas. Fortaleza, Ceará, 2007.

\begin{tabular}{lrr}
\hline \multicolumn{1}{c}{ Variáveis } & $\mathbf{n}=\mathbf{1 1}$ & $\%$ \\
\hline Higiene das mãos & & \\
$\quad$ Sim & 10 & 91,7 \\
$\quad$ Não & 01 & 8,3 \\
Explicação do procedimento & & \\
$\quad$ Sim & 08 & 72,7 \\
$\quad$ Não & 03 & 27,3 \\
Explicação dos efeitos pós-vacinais & & \\
$\quad$ Sim & 03 & 27,3 \\
Não & 08 & 72,7 \\
\hline
\end{tabular}

Fonte: Pesquisa direta, 2007.

trica. Os termômetros digitais, ou cabo extensor, que medem as temperaturas do momento, máxima e mínima estavam todos funcionando, alguns danificados aparentemente, e as temperaturas evidenciadas não condiziam com as recomendações do PNI ${ }^{27}$.

Em relação à estrutura física, $41,7 \%$ não tinham bancadas, armários sobre e sob a bancada, como sugerido pelo manual da estrutura física das salas de vacina ${ }^{26}$, contudo eram localiza- das de acordo com os padrões do PNI no que diz respeito ao fluxo dos usuários ${ }^{26}$. Sobre a decoração do ambiente, em 91,3\% havia desenhos infantis e cartazes informativos afixados nas paredes. Quanto às condições climáticas, 41,7\% eram climatizadas e as demais ventiladas artificialmente. Em 75,0\% das unidades $(n=11)$, o mapa diário de registro da temperatura estava preenchido com valores que o PNI contesta, ou seja, valores mínimos de $0,9^{\circ} \mathrm{C}$ e valores máximos de até $18,9^{\circ}$ C. Embora em todas as salas a conservação das vacinas fora da geladeira se desse em caixas térmicas de poliuretano, não se constatou o controle de temperatura dessas caixas.

\section{Discussão}

Os profissionais de enfermagem são responsáveis pelas imunizações, como justifica a literatu$\mathrm{ra}^{1,3,19,20}$; não obstante, a lei do exercício profissional brasileira permite a administração de vacinas por profissionais de enfermagem, médicos e farmacêuticos ${ }^{3}$.

Sobre a faixa etária de profissionais que trabalham em salas de vacina, este estudo é corroborado por pesquisas realizadas com equipes de enfermagem no sentido de identificar o conhecimento e o cumprimento das recomendações téc- 
nicas do PNI sobre a conservação e a aplicação dos imunobiológicos em Unidades Básicas de Saúde, envolvendo dois estados da região Sudes$\mathrm{te}^{3,19,20}$ e uma capital da região Nordeste $\mathrm{N}^{20}$. Outros dados que se mantêm semelhantes a essa literatura ${ }^{3,19,20}$ dizem respeito à experiência de trabalho e a treinamentos realizados em vacinação entre esses profissionais.

Entende-se que as atualizações sistemáticas em vacinação são uma exigência para se oferecer um serviço eficaz que erradique e controle as doenças imunopreveníveis ${ }^{6}$. Em virtude da expansão dos programas nacionais de imunização ${ }^{9}$, em nível mundial ${ }^{10}$, e da rápida inclusão de novas vacinas e recomendações ${ }^{8}$, torna-se imprescindível instrumentalizar os profissionais responsáveis por essa demanda em saúde. Neste estudo, muitas das profissionais de enfermagem referiram que não participaram de treinamento no último ano, podendo-se inferir que a falta de atualização influencia a dinâmica do serviço dos profissionais que atuam em atividades de imunização nas unidades de saúde e vai de encontro aos avanços científicos e tecnológicos nessa área ${ }^{5}$.

O Ministério da Saúde preconiza que sejam disponibilizados nas salas de vacinas os manuais, resoluções e informes técnicos operacionais para prováveis consultas pelos profissionais, assegurando a realização de procedimentos e informações, favorecendo a minimização de complicações e possibilitando educação em saúde entre os usuários ${ }^{6}$. Neste estudo, houve divergências entre enfermeiras e técnicas/auxiliares quanto à disponibilidade de literatura de atualização (manuais) e consultas on-line.

Entre as unidades básicas de saúde avaliadas, o estudo mostrou que as salas de vacina não estão com sua estrutura física adequada conforme recomendado pelo Manual de estrutura física das unidades básicas de saúde ${ }^{26}$, como evitar luz solar incidente e ter área mínima de nove metros quadrados, com dimensão mínima de 2,50 m. Em contrapartida, a maioria das salas estava bem localizada, pois o fluxo de pacientes para elas é feito de modo que o usuário não transite nas demais dependências da UBS, como recomenda o Ministério da Saúde ${ }^{26}$.

A Fundação Nacional de Saúde, em 2001, preconizou que nas salas de vacinação é importante que os procedimentos desenvolvidos garantam a máxima segurança, prevenindo infecções nas crianças e nos adultos atendidos. Para isso, as instalações devem ter paredes e pisos laváveis; pia e interruptores para uso exclusivo de cada equipamento elétrico; ser arejadas e bem ilumi- nadas, evitando-se, porém, a incidência de luz solar direta. Não é recomendado o uso de pisos de madeira, carpetes e cortinas, pois esses materiais favorecem proliferação de fungos e outros microrganismos ${ }^{26}$.

Nesta pesquisa, nem todos os profissionais preencheram o mapa de temperatura, porém a Fundação Nacional de Saúde ${ }^{27}$ preconiza que o controle diário de temperatura dos equipamentos da rede de frio é imprescindível em todas as instâncias de armazenamento para assegurar a qualidade dos imunobiológicos. Estudo realizado no Brasil descreve falhas no cumprimento das recomendações para a conservação, necessidade de supervisão permanente e adequação dos recursos humanos e equipamentos. Também há relatos de avaliações de amostras de vacinas coletadas nas unidades de saúde com comprometimento na potência ${ }^{3}$.

Os registros de temperatura encontrados neste estudo mostraram temperaturas máximas, mínimas e do momento, porém com valores acima de $+8,0^{\circ} \mathrm{C}$ e abaixo de $+2,0^{\circ} \mathrm{C}$, comprometendo a qualidade dos imunobiológicos ofertados à população $0^{6,27} \mathrm{e}$ as recomendações técnicas do Ministério da Saúde ${ }^{27}$, segundo as quais a temperatura não deve situar-se entre $+2 \mathrm{e}+8^{\circ} \mathrm{C}$ em todas as instâncias de armazenamento para que se possa assegurar a sua qualidade, sendo imprescindível o controle diário de temperatura.

A temperatura em Fortaleza registra uma média de $28^{\circ} \mathrm{C}$, sendo importante amenizar a temperatura ambiente das salas de vacinas. Mantendo a temperatura elevada, mais rápido a temperatura do interior da caixa térmica aumenta pela entrada de ar quente pelas paredes da cai$\mathrm{xa}^{27}$. Para a preservação dos imunobiológicos não é normativo que as salas de vacinas sejam climatizadas, sendo necessário que obedeçam às regras de conservação ${ }^{27}$.

O MS considera que a garantia da qualidade dos imunobiológicos, desde a sua produção até o momento em que eles são administrados, é uma constante preocupação daqueles que manuseiam esses produtos. Assegurar a conservação adequada não está relacionado apenas à questão da perda financeira, mas principalmente ao risco de possível desabastecimento, sendo que o objetivo da rede de frio é assegurar que todos os imunobiológicos mantenham as suas características iniciais, para conferir imunidade ao indivíduo vacinado ${ }^{27}$.

É importante mencionar que achados incongruentes na conservação das vacinas também foram encontrados em outros estudos ${ }^{19,20}$ no 
Brasil e em outros países ${ }^{5}$.

Neste trabalho, identificou-se que ainda existe profissional que não aderiu à higienização das mãos, ao realizar o procedimento. Canela e Coelho ${ }^{28}$ identificaram que a limpeza usualmente é suficiente para remover a microbiota transitória humana, e a técnica recomendada é a higiene simples com água e sabão ou detergentes sintéticos, sob fricção, para remover sujidade, células epiteliais mortas, resíduos do metabolismo que constituem o substrato para os microrganismos. Essa higienização, se adotada pelos profissionais da saúde, confere um padrão de segurança para a maioria dos procedimentos que não requer técnica asséptica. Diante disso, a lavagem das mãos deve ser um hábito no cotidiano dos profissionais de saúde; todavia, a sua adesão e continuidade desafiam os profissionais do controle de infecção hospitalar ${ }^{29}$.

O cuidado centrado no usuário demanda que o profissional oriente os procedimentos a serem executados, assim como a ocorrência de possíveis reações adversas pós-vacinais, evitando-se notícias distorcidas que possam afastar a comunidade das vacinações sistemáticas e diminuir as coberturas vacinais ${ }^{6}$. Neste trabalho, os profissionais de outras categorias de saúde encaminham o usuário para a sala de vacina, sendo essa atitude significativa para o aumento da cobertura vacinal da população.

Uma das causas das baixas coberturas de vacinação é a perda de oportunidades para vacinar crianças. O trabalho das equipes de saúde com a população e as organizações da comunidade permite melhor aproveitar as oportunidades. Para superar as perdas, uma tarefa essencial é procurar sensibilizar todos os profissionais da equipe para que se envolvam na atividade de vacinação $0^{6,30-32}$.

Nesse sentido, a busca ativa de faltosos é uma das atribuições determinada pela Estratégia Saúde da Família. As ações dos agentes comunitários de saúde e as equipes de saúde da família tiveram reflexos diretos no Programa Nacional de Imunizações. A reorganização dos serviços e a efetiva imunização aumentaram a cobertura vacínica em crianças menores de um ano ${ }^{16}$.

A pesquisa permitiu verificar que em algumas unidades a sala de vacina não é exclusiva para esta ação. A literatura ${ }^{33}$ reafirma não ser permitida a realização de outros procedimentos nesse setor. Contudo, o manual de estrutura física das unidades básicas de saúde da família considera que nas salas de vacinas podem ser administrados medicamentos injetáveis ${ }^{26}$.

A imunização é um forte instrumento de pro- teção à saúde e requer estudos frequentes, pois as vacinas disponíveis são eficazes e seguras, desde que sejam obedecidos todos os aspectos técnicos e operacionais na sala de vacinas. Os aspectos operacionais em sala de vacina merecem uma atenção especial, pois tratam de medidas essenciais para a aplicação de imunobiológicos dentro de todos os padrões corretos de conservação, armazenagem e indicações clínicas ${ }^{33}$.

Os custos com essas vacinas têm aumentado consideravelmente nos últimos anos, principalmente pelo surgimento de novas vacinas no calendário básico do programa de imunização. Desse modo, eleger a utilização de vacinas como o mais bem-sucedido instrumento no âmbito dos trabalhos pertencentes à saúde pública significa um exemplo digno de ser imitado, prestigiado e $\operatorname{ampliado}^{34,35}$.

\section{Conclusão}

O estudo evidenciou que ter experiência na atenção básica e em salas de vacina e estar atualizado sobre o tema mostrou percentual significativo entre as participantes do estudo. Pelo dinamismo que as atividades de vacinação demandam desde a aquisição dos imunobiológicos, acondicionamento, conservação, manuseio, administração e orientações abrangentes, o gerenciamento desse setor exige um profissional apto a acompanhar a evolução científica e tecnológica dos imunobiológicos.

As condições de trabalho em relação aos procedimentos e atividades diárias ofertadas à população-alvo, na visão das participantes, favorecem um trabalho de qualidade, enquanto a estrutura física e os equipamentos necessários para cumprir as recomendações técnicas do PNI mostraram fragilidades.

Estudiosos do tema sinalizam que é importante refletir sobre a sucessão de falhas que podem comprometer a credibilidade que os imunobiológicos vêm conquistando nas últimas décadas, tornando-se imperativo para se manter essa confiança a orientação dos profissionais e o monitoramento dos processos que envolvem a manipulação dessas substâncias, por parte dos supervisores das unidades e gestores de saúde dos municípios ${ }^{20}$. Igualmente, é indiscutível a importância da manutenção da rede de frio para assegurar que as vacinas mantenham suas características imunogênicas desde a produção até a administração da vacina à comunidade ${ }^{19}$.

Importante mencionar que o estudo aponta 
para a necessidade da educação permanente na formação dos profissionais responsáveis pelas imunizações, e nesse sentido a equipe de enfermagem assegura a qualidade da imunização ofertada à população. Ainda pode-se recomendar, a partir desta pesquisa, a necessidade de ampliar investigações sobre o processo de trabalho em salas de vacinas, incluindo os âmbitos público e privado, que ofertam esse serviço relevante para a manutenção da saúde entre os povos.

\section{Colaboradores}

GLM Luna e LJES Vieira orientaram todas as etapas do trabalho, análise dos dados e redação final do artigo; PF Souza foi autora da concepção teórica, coleta, análise preliminar dos dados e redação inicial do texto; SVG Lira, DP Moreira e AS Pereira colaboraram na atualização da revisão de literatura, na discussão dos dados e na revisão crítica do texto.

\section{Agradecimentos}

À Coordenação da Atenção Básica da Secretaria de Saúde do Município de Fortaleza, aos gestores dos Centros de Saúde da Família da Secretaria Executiva Regional IV e aos profissionais da equipe de enfermagem das salas de vacinas das unidades participantes do estudo.

\section{Referências}

1. Mitchell AD, Bossert TJ, Yip W, Mollahaliloglu S. Health worker densities and immunization coverage in Turkey: a panel data analysis. Human Resources for Health [periodico na Internet] 2008 [acessado 2010 mar 12]; 6:[cerca de 22 p.]. Disponível em: http://www.human-resources-health.com/content/6/ $1 / 29$

2. Jani JV, De Schacht C, Jani IV, Bjune G. Risk factors for incomplete vaccination and missed opportunity for immunization in rural Mozambique. BMC Public Health [periódico na Internet] 2008 [acessado 2010 mar 12]; (8) [9p.] Disponível em: http:// www.biomed central.com

3. Aranda CMSS, Moraes JC. Rede de frio para a conservação de vacinas em unidades públicas do município de São Paulo: conhecimento e prática. Rev Bras Epidemiol 2006; 9(2):172-185.

4. Cunha J, Krebs LS. Imunizações. In: Duncan BB, Schmidt MI, Giugliani ERJ. Medicina ambulatorial: condutas clínicas em Atenção Primária. $3^{\mathrm{a}}$ ed. Porto Alegre: Artes Médicas; 2006. p. 1295-1307.

5. National Center for Immunization and Respiratory Diseases, Centers for Disease Control and Prevention. Epidemiology and prevention of vaccine-preventable diseases. $11^{\text {th }}$ ed. [acessado 2010 mar 12]. Disponível em: http://www.cdc.gov/vaccines/pubs/ pinkbook/default.htm

6. Brasil. Ministério da Saúde. Manual de normas de vacinação. $3^{a}$ ed. Brasília: Ministério da Saúde: Fundação Nacional de Saúde; 2001.

7. Feijó RB, Sáfadi MA. Imunizações: três séculos de uma história de sucesso e constantes desafios. J Pediatr 2006; 82(3):S1-S3.

8. Brasil. Ministério da Saúde. [site da Internet]. 2010 [acessado 2010 mar 10]. Disponível em: http:// portal.saude.gov.br/portal/aplicacoes/noticias/ default.cfm?pg=dspDetalheNoticia\&id_area $=124 \& C O$ _ NOTICIA $=1108$

9. Brasil. Informe técnico operacional: estratégia nacional de vacinação contra o vírus influenza pandêmica (H1N1). Brasília: Ministério da Saúde; 2010.

10. Center for Disease Control and Prevention (CDC). H1N1. O que oce ustad saber (Espanhol). [acessado 2010 mar 10]. Disponível em: http://www.immunize org/vis/sp_h1n1_live.pdf 
11. Brasil. Nota técnica referente à vacinação de gestantes contra hepatite B na rede do SUS. [acessado 2010 mar 10]. Disponível em: http://portal.saude.gov.br/portal/arquivos/pdf/ nota_tecnica_hepatite007.pdf

12. Rio Grande do Sul. Implementação da vacinação contra hepatite B em manicures, podólogos, barbeiros e tatuadores. [acessado 2010 marc 10]. Disponível em: http://www.saude.rs.gov.br/dados/12526961346 171228750140697nota_tecnica_vacinacao_manicures \%2520hepatite\%2520b_2008.pdf

13. Starfield B. Atenção Primária: equilíbrio entre necessidades de saúde, serviços e tecnologia. Brasília: Unesco/Ministério da Saúde; 2002.

14. Canesqui AM, Oliveira AMF. Saúde da Família: modelos internacionais e estratégia brasileira. In: Negri B, Viana ALA, organizadores. O Sistema Único de Saúde em dez anos de desafio: o passo a passo de uma reforma que alarga o desenvolvimento e estreita a desigualdade social. São Paulo: Sobravine \& Cealag; 2002. p. 241-269.

15. Milennium Project. Commissioned by the UN Secretary General and supported by the UN Development Group. [acessado 2010 mar 10]. Disponivel em: http:/ /www.pnud.org.br/milenio/ft4.php

16. Brasil. Regionalização da assistência à saúde: aprofundando a descentralização com equidade no acesso. Norma Operacional da Assistência à Saúde - NoasSUS 01/02. Brasília; Ministério da Saúde; 2002.

17. Souza HM. Saúde da Família: desafios e conquistas. In: Negri B, Viana ALA, organizadores. O Sistema Único de Saúde em dez anos de desafio: o passo a passo de uma reforma que alarga o desenvolvimento e estreita a desigualdade social. São Paulo: Sobravine \& Cealag; 2002. p. 221-240.

18. Brasil. Portaria $n^{\circ} 325 / G M$, de 21 de fevereiro de 2008. Brasília: Ministério da Saúde; 2008.

19. Oliveira VC, Guimarães EAA, Guimarães IA, Januário LH, Ponto IC. Prática da enfermagem na conservação de vacinas. Acta Paul Enferm 2009; 22(6):814-818.

20. Melo GKM, Oliveira JV, Andrade MS. Aspectos relacionados à conservação de vacina nas unidades básicas de saúde da cidade do Recife - Pernambuco. Epidemiol Serv Saúde 2010; 19(1):25-32.

21. Instituto Brasileiro de Geografia e Estatística (IBGE). [acessado 2010 mar 10]. Disponível em: http:// www.ibge.gov.br/cidadesat/topwindow.htm?1

22. Datasus. Indicadores de Saúde. Mortalidade Infantil. [acessado 2010 mar 10]. Disponível em: http:// tabnet.datasus.gov.br/cgi/idb2008/matriz.htm? saude $=$ http $\% 3 \mathrm{~A} \% 2 \mathrm{~F} \% 2 \mathrm{Fp}$ ortal.saude.gov.br $\% 2 \mathrm{~F}$ portal\%2Fsaude\%2Farea.cfm\%3Fid_area\%3D165\& botaook=OK\&obj=http\%3A\%2F\%2Ftabnet.datasus. gov.br\%2Fcgi\%2Fidb2008\%2F matriz.htm\#mort

23. Relatório o Estado das Cidades do Mundo 2010/2011. [acessado 2010 mar 10]. Disponível em: http:// www.oeco.com.br/blog-ecocidades/106-blog-ecocidades/23681-o-estado-das-cidades-no-mundo

24. Ceará. Fortaleza. Prefeitura Municipal de Saúde. [site na Internet] [acessado 2010 mar 10]. Disponível em: http://www.fortaleza. ce.gov.br/

25. Brasil. Manual de eventos adversos pós-vacinação. Brasília: Ministério da Saúde; 2005.
26. Brasil. Departamento de Atenção Básica. Manual de estrutura física das unidades básicas de saúde: saúde da família. 2a ed. Brasília: Ministério da Saúde; 2008. (Série A. Normas e Manuais Técnicos).

27. Brasil. Ministério da Saúde. Manual da rede de frio. Brasília: Ministério da Saúde; 2007.

28. Canela LCGCS, Coelho MJ. Lavagem das mãos como um cuidado essencial no ambiente hospitalar: de Florence Nightingale e Ignaz Semmelweis ao milênio atual (1847-2003). Enfermagem Atual 2003; 3(1):11-16.

29. Brasil. Agência Nacional de Vigilância Sanitária. Higienização das mãos em serviços de saúde. Brasília: Agência Nacional de Vigilância Sanitária; 2007.

30. Tertuliano GC, Stein AT. Atraso vacinal e seus determinantes: um estudo em localidade atendida pela Estratégia Saúde Família. [acessado 2010 mar 10]. Disponível em: http://www. abrasco.org.br/cienciaesaudecoletiva/artigos/artigo_int.php?id_artigo $=2172$

31. Corluka A, Walker DG, Lewin S, Glenton C, Scheel IB. Are vaccination programmes delivered by lay health workers cost-effective? A systematic review. Human Resources for Health [periódico na Internet]. 2009 [acessado 2010 mar 10]; (7): [20 p.]. Disponível em: http://www.human-resources-health. $\mathrm{com} /$ content $/ 7 / 1 / 81$

32. Benzekri N, Goldman E, Lewis F, Johnson CC, Reynolds SM, Reynolds MG, Damon IK. Laboratory worker knowledge, attitudes and practice towards smallpox vaccine. Occupational Medicine 2010 [acessado 2010 mar 10]; 60 [4p.]. Disponível em: http:// occmed.oxfordjournals.org/cgi/content/short/60/1/75

33. Zamberlan AGON. Aspectos operacionais na sala de vacina. Botucatu, SP: Hospital das Clínicas da Faculdade de Medicina de Botucatu (Unesp); 2007. [acessado 2010 mar 10]. Disponível em: http:// www.vacinas.org.br/novo/aspectos_operacionais/ na_sala_de_vacinas.htm

34. Amato NV, Pasternak J. Influenzas sim, gripe não. Rev Soc Bras Med Trop [on-line] 2000; 33(5):499-500. [acessado 2007 abr]. Disponível em: http:// scielo.br/pdf/rsbmt/v33n5/3131.pdf

35. Vilarino MAM, Lopes MJM, Bueno ALM, Brito MRV. Idosos vacinados e não vacinados contra a influenza: morbidade relatada e aspectos sociodemográficos, Porto Alegre (RS, Brasil), 2004. Cien Saude Colet 2010; 15(6):2879-2886.

Artigo apresentado em 31/3/2010

Aprovado em 30/9/2010

Versão final apresentada em 6/10/2010 